\title{
Tuberculose em Idade Pediátrica: Experiência de 12 Anos num Centro Terciário de Referência em Portugal
}

\author{
Pediatric Tuberculosis: 12 Years of Experience in a Tertiary \\ Referral Center in Portugal
}

Madalena BORGES ${ }^{1}$, Ana Paula ROCHA ${ }^{1}$, Carlota VEIGA DE MACEDO ${ }^{1}$, Tiago MILHEIRO SILVA ${ }^{1}$,
Catarina GOUVEIA ${ }^{1}$, Flora CANDEIAS ${ }^{1}$, Maria João BRITO ${ }^{1}$

Acta Med Port 2022 May;35(5):367-375 - https://doi.org/10.20344/amp.16474

RESUMO

Introdução: Em Pediatria, o diagnóstico de tuberculose constitui um desafio, pois a doença pode frequentemente manifestar-se através de formas graves e extrapulmonares. O objetivo deste estudo foi avaliar e refletir sobre a tuberculose grave com necessidade de internamento, em idade pediátrica, numa área metropolitana de Lisboa.

Material e Métodos: Estudo descritivo de doentes com idade inferior a 18 anos, internados com o diagnóstico de tuberculose num hospital pediátrico terciário, de 2008 a 2019 (12 anos).

Resultados: Identificados 145 doentes, numa média de 12 casos por ano, e um aumento do número de casos nos últimos três anos. A mediana de idades dos doentes era de 12,9 anos, 42,8\% nascidos em países africanos de língua oficial portuguesa e $20 \%$ tinham doença crónica. Diagnosticou-se tuberculose pulmonar em 52,4\% $(n=76)$ e tuberculose extrapulmonar em 47,6\%: ganglionar ( $n=26)$, óssea $(n=15)$, miliar $(n=8)$, meníngea $(n=7)$, peritoneal/intestinal $(n=6)$, pleural $(n=4)$, renal $(n=1)$, cutânea $(n=1)$, da parede torácica $(n=1)$ e glândulas salivares $(n=1)$. A prova tuberculínica foi positiva em $78 / 99(78,8 \%)$ e o interferon gamma release assay em $61 / 90(67,8 \%)$. Em 20,7\% ( $n=30)$ identificaram-se bacilos ácido-álcool resistentes no exame direto do suco gástrico/expetoração e o agente foi identificado em $59,3 \%(n=86)$. A tuberculose resistente ocorreu em $11 \%(n=16)$. Os doentes com tuberculose extrapulmonar eram mais jovens $(p=0,006)$ e fizeram tratamentos mais prolongados $(p<0,001)$. Ocorreram complicações da terapêutica em $11 \%(n=16)$. Registou-se um óbito numa doente com neoplasia avançada.

Conclusão: Este estudo alerta para a necessidade do rastreio da infeção em crianças de países endémicos, imunossuprimidos e com doença crónica.

Palavras-chave: Criança; Tuberculose; Tuberculose Pulmonar

\section{ABSTRACT}

Introduction: The diagnosis of tuberculosis in children is a challenge namely because extrapulmonary tuberculosis and severe disease are more frequent in this age group. The aim of this study was to evaluate and reflect about severe tuberculosis in pediatric age, in a metropolitan area of Lisbon.

Material and Methods: Descriptive study about patients under 18 years of age admitted with tuberculosis disease in a tertiary pediatric hospital, from 2008 to 2019 (12 years).

Results: We report 145 patients, average of 12 cases/year, with an increase in the last three years. Median age of 12.9 years, $42.8 \%$ born in Portuguese-speaking African countries and $20 \%$ had a chronic disease. The diagnosis was pulmonary tuberculosis in $52.4 \%$ $(n=76)$ and extrapulmonary tuberculosis in $47.6 \%$ : lymphatic $(n=26)$, skeletal $(n=15)$, miliary $(n=8)$, meningeal $(n=7)$, peritoneal/ intestinal $(n=6)$, pleural $(n=4)$, renal $(n=1)$, cutaneous $(n=1)$, thoracic wall $(n=1)$ and salivary glands $(n=1)$. The tuberculin test was positive in 78/99 (78.8\%) and interferon gamma release assay in $61 / 90(67.8 \%)$. In $20.7 \%(n=30)$ acid-fast bacilli were identified in gastric aspirate/sputum and the agent was identified in $59.3 \%(n=86)$. Tuberculosis was resistant in $11 \%(n=16)$. Patients with extrapulmonary tuberculosis were younger $(p=0.006)$ and had more prolonged therapy $(p<0.001)$. Therapy-related complications occurred in $11 \%(n=16)$. One patient died (with terminal cancer).

Conclusion: This study highlights the need for screening of tuberculosis in children from endemic countries, patients with immunosuppression and chronic disease.

Keywords: Child; Tuberculosis; Tuberculosis, Pulmonary

\section{INTRODUÇÃO}

Em 2017, a Organização Mundial de Saúde (OMS) estimou a existência de 10 milhões de novos casos de tuberculose (TB) em todo o mundo e 1,3 milhões de mortes atribuíveis a esta patologia. ${ }^{1} \mathrm{~A}$ incidência em países desenvolvidos é inferior a 10 casos por 100000 habitantes, mas pode atingir 150 a 500 casos por 100000 habitantes em países endémicos. Neste mesmo ano, um milhão de crianças desenvolveu TB. ${ }^{1}$ Em 2018 e 2019, o número de novos casos manteve-se estável entre 9 e 11,1 milhões em todo o mundo, sendo $11 \%$ e $12 \%$ em crianças com menos de 15 anos de idade, respetivamente..$^{2,3}$

Em Portugal, as taxas de incidência de TB em 2014 eram inferiores a 20 casos por 100000 habitantes, colocando o país no grupo de baixa incidência para a doença. Assim, e de acordo com as recomendações da OMS para os países com baixa incidência, sistemas eficazes de vigilância e taxas de incidência de meningite tuberculosa inferiores a um caso por 10000000 habitantes durante cinco anos consecutivos, foi implementada, a partir de junho de 2016, a vacinação com bacille Calmette-Guérin (BCG),

\footnotetext{
1. Unidade de Infeciologia. Área de Pediatria. Hospital Dona Estefânia. Centro Hospitalar Universitário de Lisboa Central. Lisboa. Portugal.

$\square$ Autor correspondente: Madalena Borges. madalenalmeidaborges@gmail.com

Recebido/Received: 28/04/2021 - Aceite/Accepted: 12/07/2021 - Publicado Online/Published Online: 06/01/2022 - Publicado/Published: 02/05/2022 Copyright (C) Ordem dos Médicos 2022
} 
apenas para grupos de risco (Tabela 1). ${ }^{4}$

Nesse mesmo ano de 2014, as áreas metropolitanas do Porto, Lisboa, Setúbal e Algarve tiveram elevadas taxas de incidência ( $>20$ casos e $<50$ casos/100 000 habitantes) ${ }^{5}$ Mesmo em 2016, quando a taxa de incidência nacional foi de 18 casos por 100000 habitantes, continuou a verificar-se uma concentração de casos nos distritos de Lisboa e do Porto, evidenciando uma distribuição da doença em bolsas no nosso país. ${ }^{6}$

Simultaneamente, embora a incidência da TB em imigrantes de outros países seja 4,8 vezes superior à incidência nacional estimada $(95,4$ casos por 100000 habitantes em 2014 e 86,7 casos por 100000 habitantes em 2016) a percentagem de doentes imigrados com TB manteve-se estável com $15,9 \%$ e $18,4 \%$ nesses anos. ${ }^{5,6}$

A TB é causada pelo Mycobacterium tuberculosis, um bacilo aeróbio, ácido-álcool resistente, transmitido por via aérea. As partículas inaladas atingem os gânglios mediastínicos e vias aéreas terminais, formando-se o complexo de Ghon, que inclui o foco inicial da infeção e a linfadenopatia regional. Nesta fase, a infeção pode ficar contida, disseminar-se ou posteriormente ocorrer reativação. Assim a evolução inclui três formas distintas. A tuberculose latente ou infeção, que ocorre num indivíduo assintomático com radiografia de tórax normal, mas que evidencia contacto com o bacilo, com prova tuberculínica e/ou interferon gamma release assay (IGRA) positivo. A doença pulmonar com linfadenopatias intratorácicas e doença parenquimatosa ocorre na presença de sinais e sintomas compatíveis e/ou alterações na radiografia de tórax. Neste caso, quando o bacilo é identificado por exame direto na expetoração ou suco gástrico, considera-se que o doente é contagioso. ${ }^{7} \mathrm{Na}$ forma tuberculose extrapulmonar, a mais comum é a ganglionar $(67 \%)$ seguida de outras mais raras como a meníngea $(13 \%)$, pleural $(6 \%)$, miliar $(5 \%)$ e óssea $(4 \%) .8$

Em Pediatria, ao contrário do adulto, a maioria das infeções evoluem para doença com o aparecimento de manifestações clínicas, um a dois anos após a infeção inicial. ${ }^{9,10}$ Em crianças com menos de dois anos de idade, a evolução para doença ocorre em $10 \%$ - $40 \%$ dos casos, ${ }^{9}$ sendo também maior o risco de doença disseminada e meníngea ( $2 \%$ - 20\%). ${ }^{11}$

Em países desenvolvidos, a TB é atualmente considerada uma entidade rara, mas continua a ser um importante problema de saúde em todo o mundo, exigindo um esforço contínuo no desenvolvimento de estratégias para o seu controlo. ${ }^{1}$ Foi nosso objetivo avaliar e refletir sobre a doença no doente com TB grave e necessidade de internamento, na idade pediátrica, numa área metropolitana de Lisboa.

\section{MATERIAL E MÉTODOS}

Estudo observacional e descritivo em doentes até aos 18 anos de idade internados num hospital terciário com diagnóstico de TB, durante 12 anos (entre janeiro de 2008 e dezembro de 2019). Foram analisados dados sociodemográficos, epidemiológicos, vacinação com BCG, clínica, exames complementares, terapêutica e complicações.

Os critérios para positividade do teste tuberculínico foram $\geq 5 \mathrm{~mm}$ em não vacinados e imunodeprimidos, e $\geq 10$ $\mathrm{mm}$ em vacinados e ainda a presença de flictenas ou ulceração independentemente do estado vacinal.

A análise estatística foi realizada com recurso a Statistical Package for Social Sciences ${ }^{\circledR}$ (SPSS) versão 23.0. Para verificar a normalidade das variáveis contínuas foi utilizado o teste de Shapiro-Wilk. Para comparar o comportamento das variáveis contínuas com distribuição normal entre os grupos de doentes com tuberculose pulmonar (TP) e tuberculose extrapulmonar (TbEP) foi utilizado o teste $t$-Student; nas variáveis contínuas que não verificaram a distribuição normal foi utilizado o teste não paramétrico de Mann-Whitney. Para as variáveis nominais, a comparação dos diferentes grupos definidos (TP versus TbEP) foi efetuada pela aplicação do teste de qui-quadrado. Na aplicação dos testes de hipóteses foi considerado um nível de significância de $5 \%$.

Este estudo foi aprovado pela Comissão de Ética do Hospital D. Estefânia, em Lisboa.

Tabela 1 - Grupos de risco para tuberculose ${ }^{4}$

\begin{tabular}{ll}
\hline Crianças sem registo de BCG*I & Situações abrangidas: \\
Sem cicatriz vacinal e: & - Países com incidência de tuberculose $\geq 40 / 100$ 000 \\
\hline Provenientes de países com elevada & - Estadia de pelo menos, 3 meses \\
incidência de tuberculose & A avaliar pelas unidades de Saúde Pública em articulação com os coordenadores \\
Que terminaram o processo de rastreio de & regionais do Programa Nacional para a Tuberculose e CDP** \\
contactos e/ou esquema de profilaxia & - Infeção VIH/SIDA, após exclusão de infeção VIH na criança (se mãe VIH positiva) \\
Cujos pais, outros coabitantes ou & - Dependência de álcool ou de drogas \\
conviventes apresentem & - Proveniência de país com elevada incidência de tuberculose \\
& - Antecedentes de tuberculose \\
Pertencentes a comunidades com risco & A avaliar pelas unidades de Saúde Pública em articulação com os coordenadores \\
elevado de tuberculose & regionais do Programa Nacional para a Tuberculose e CDP \\
Viajantes para países com elevada & - Países com incidência de tuberculose $\geq 40 / 100$ 000 \\
incidência de tuberculose & - Estadia de, pelo menos, 3 meses \\
& - Pode ser ponderada a vacinação para estadias mais curtas, se for considerado \\
& um elevado risco de infeção \\
\hline
\end{tabular}

* BCG: Bacillus Calmette-Guérin; ** CDP: centros de diagnóstico pneumológico

Adaptado de: Direção-Geral da Saúde. Estratégia de vacinação contra a tuberculose com a vacina BCG, NORMA Nº 006/2016. 2016. 


\section{RESULTADOS}

Identificámos um total de 145 doentes, com uma média de 12 casos por ano e aumento do número de casos nos últimos três anos $(n=66 ; 45,5 \%)$ (Fig. 1$)$ à custa da população portuguesa (Fig. 2) com um pico em 2017 (12 portugueses/22 casos) e 2019 (15 portugueses/23 casos).

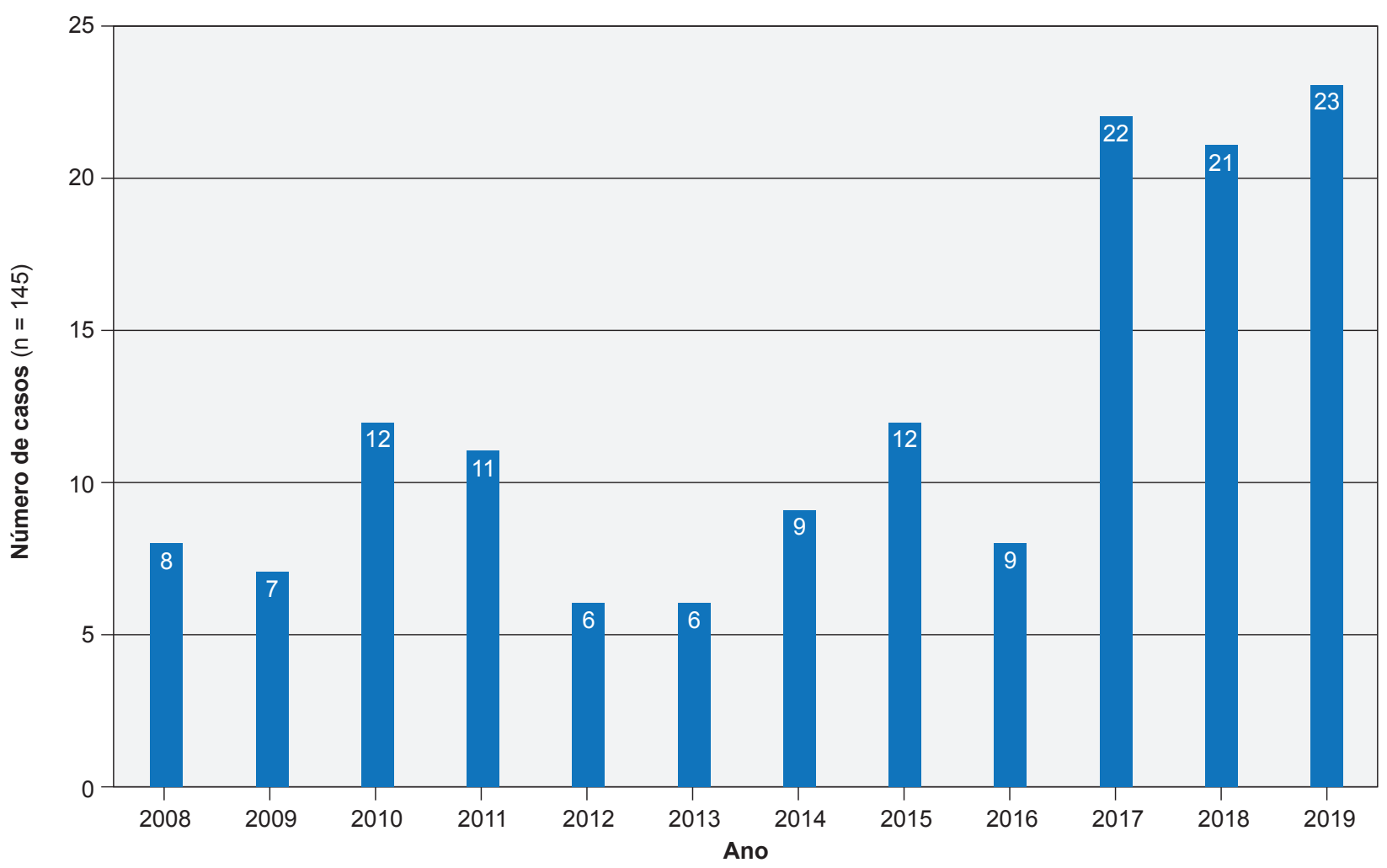

Figura 1 - Distribuição dos casos de tuberculose diagnosticados ao longo dos anos

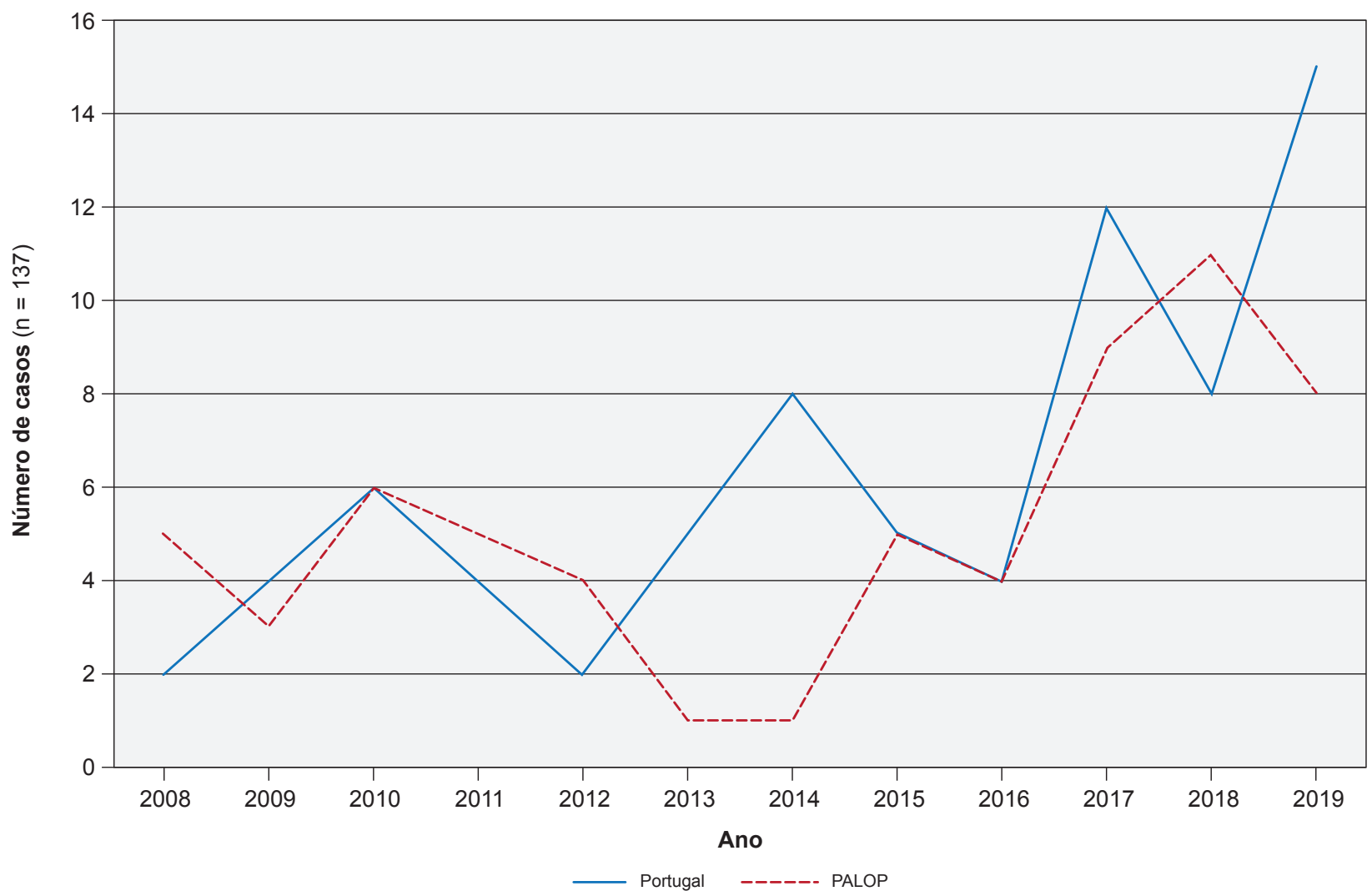

Figura 2 - Distribuição dos casos de tuberculose entre os doentes naturais de Portugal e de PALOP 
Nos primeiros nove anos 50,6\% (40/79) dos doentes eram portugueses, 43\% (34/79) eram naturais dos PALOP (Países Africanos de Língua Oficial Portuguesa), 2,5\% (2/79) da Ásia, 2,5\% (2/79) da América do Sul e 1,3\% (1/79) do Leste Europeu. Nos últimos três anos, $53 \%$ dos doentes $(35 / 66)$ eram portugueses, $42,4 \%(28 / 66)$ eram naturais dos PALOP e 4,5\% (3/66) da Ásia.

A caracterização da população pediátrica com TB é apresentada na Tabela 2.

Registou-se um ligeiro predomínio do género masculino (54,5\%), com uma mediana de idades de 12,9 anos [3,916,1 anos]. A maioria, $60 \%(n=87)$, tinha mais de 10 anos, $36,6 \%(n=53)$ mais de 15 anos de idade e $7,6 \%(n=11)$ idade inferior ou igual a 12 meses. Mais de metade dos doentes eram naturais de Portugal $(51,7 \%)$, com $42,8 \%$ oriundos de
PALOP, registando-se ainda casos provenientes de outros países $(5,5 \%)$ : Ásia $(n=5)$, América do Sul $(n=2)$ e Leste Europeu $(n=1)$. Na altura do internamento, relativamente aos doentes de PALOP, o tempo de residência em Portugal apresentava uma mediana de 4,5 meses [0 - 15 anos].

A maioria das crianças $(74,5 \%)$ tinha realizado a vacina BCG, confirmada por informação no boletim individual de saúde e/ou cicatriz vacinal. Dos 37 doentes não vacinados, 14 eram portugueses (10 nascidos após 2015), 20 eram naturais dos PALOP e três eram de origem asiática.

Vinte e nove doentes (20\%) tinham doença crónica e dois tinham mais do que uma patologia. Duas crianças de origem africana estavam infectadas pelo vírus da imunodeficiência humana (VIH). Num caso, o diagnóstico foi realizado simultaneamente com o de TB e em outro doente

Tabela 2 - Caracterização dos doentes com tuberculose

\begin{tabular}{|c|c|c|}
\hline Características clínicas & & n (\%) \\
\hline Grupo etário & $\begin{array}{l}0-12 \text { meses } \\
1-5 \text { anos } \\
5-10 \text { anos } \\
>10 \text { anos }\end{array}$ & $\begin{array}{c}11(7,6 \%) \\
31(21,4 \%) \\
16(11,0 \%) \\
87(60,0 \%)\end{array}$ \\
\hline Nacionalidade & $\begin{array}{l}\text { Portugal } \\
\text { PALOP } \\
\text { Outros }\end{array}$ & $\begin{array}{c}75(51,7 \%) \\
62(42,8 \%) \\
8(5,5 \%)\end{array}$ \\
\hline BCG/cicatriz vacinal & $\begin{array}{l}\text { Sim } \\
\text { Não }\end{array}$ & $\begin{array}{c}108(74,5 \%) \\
37(25,5 \%)\end{array}$ \\
\hline Doença crónica & $\begin{array}{l}\text { Endócrina } \\
\text { Neurológica } \\
\text { Hematológica } \\
\text { Gastrointestinal } \\
\text { Reumatológica } \\
\text { Renal } \\
\text { Outras }\end{array}$ & $\begin{array}{l}7(4,8 \%) \\
6(4,1 \%) \\
5(3,4 \%) \\
4(2,8 \%) \\
2(1,4 \%) \\
2(1,4 \%) \\
5(3,4 \%)\end{array}$ \\
\hline Imunossupressão & & $4(2,8 \%)$ \\
\hline Tuberculose pulmonar & & $76(52,4 \%)$ \\
\hline Tuberculose extrapulmonar & & $69(47,6 \%)$ \\
\hline Formas de tuberculose extrapulmonar & $\begin{array}{l}\text { Ganglionar } \\
\text { Óssea } \\
\text { Miliar } \\
\text { Meníngea } \\
\text { Peritoneal } \\
\text { Pleural } \\
\text { Intestinal } \\
\text { Renal } \\
\text { Cutânea } \\
\text { Parede torácica } \\
\text { Glândulas salivares }\end{array}$ & $\begin{array}{c}26(37,7 \%) \\
15(21,7 \%) \\
8(11,6 \%) \\
7(10,2 \%) \\
4(5,8 \%) \\
4(5,8 \%) \\
2(2,9 \%) \\
1(1,4 \%) \\
1(1,4 \%) \\
1(1,4 \%) \\
1(1,4 \%)\end{array}$ \\
\hline TST & $\begin{array}{l}\text { Realizados } \\
\text { Positivo } \\
\text { Negativo } \\
\text { Anérgico }\end{array}$ & $\begin{array}{c}99 / 145(68,3 \%) \\
78 / 99(78,8 \%) \\
2 / 99(2,0 \%) \\
19 / 99(19,2 \%)\end{array}$ \\
\hline IGRA Quantiferon & $\begin{array}{l}\text { Realizados } \\
\text { Positivo } \\
\text { Negativo } \\
\text { Indeterminado }\end{array}$ & $\begin{array}{c}88 / 145(60,7 \%) \\
52 / 88(59,1 \%) \\
26 / 88(29,5 \%) \\
10 / 88(11,4 \%)\end{array}$ \\
\hline IGRA T-SPOT & $\begin{array}{l}\text { Realizados } \\
\text { Positivo } \\
\text { Negativo }\end{array}$ & $\begin{array}{c}24 / 145(16,6 \%) \\
9 / 24(37,5 \%) \\
15 / 24(62,5 \%)\end{array}$ \\
\hline
\end{tabular}


por rastreio em consulta de seguimento. Quatro doentes $(2,8 \%)$ que realizavam terapêutica imunossupressora (corticoterapia) nunca tinham sido rastreados para TB.

Em 50 crianças identificou-se um contacto com TB ativa $(34,5 \%)$. O rastreio fora realizado apenas em 29/50 casos. Em 21 crianças, apesar do contacto ser bem conhecido, não tinha sido realizado qualquer tipo de rastreio. Em cinco, tinha sido diagnosticada TB latente, pelo que estas crianças só tinham sido medicadas com isoniazida. Na maioria dos casos (62\%), o caso índex pertencia ao agregado familiar: mãe $(n=11)$, pai $(n=8)$ e outro familiar coabitante ( $n$ =12). Em 11/50 o contacto era um familiar não coabitante. Nos restantes oito, tratava-se de amigos ou vizinhos $(n=$ $4)$, de um residente da mesma instituição $(n=3)$ e registou-se um caso de infecção nosocomial $(n=1)$. O tempo que mediou entre o diagnóstico no caso índex e o diagnóstico de TB nos nossos doentes teve uma média de 13,7 meses.

Foi diagnosticada TP em 76 doentes (52,4\%). A clínica cursou com tosse $(n=51)$, febre $(n=31)$, astenia $(n=25)$, perda ponderal $(n=24)$ e toracalgia $(n=17)$. Outras apresentações clínicas menos frequentes incluíram hemoptise $(n=6)$ e pneumonia sem resposta à antibioticoterapia convencional $(n=2)$.

Registaram-se 69 casos $(47,6 \%)$ de TbEP com forma ganglionar $(n=26)$, óssea $(n=15)$, miliar $(n=8)$, meníngea $(n=7)$, peritoneal $(n=4)$, pleural $(n=4)$, intestinal $(n=2)$, renal $(n=1)$, cutânea $(n=1)$, da parede torácica $(n=1)$, das glândulas salivares $(n=1)$. Um doente apresentava simultaneamente TB óssea e pleural. A sintomatologia va- riou de acordo com a forma clínica, mas o sintoma mais frequente foi febre (52,2\% dos casos). A sintomatologia na TbEP encontra-se caracterizada na Tabela 3. A TB ganglionar cursou com febre $(n=11)$, tosse $(n=10)$, astenia $(n=$ $7)$, perda ponderal $(n=7)$, adenopatia cervical de evolução subaguda/crónica $(n=6)$ e toracalgia $(n=4)$. A tuberculose óssea também com febre $(n=8)$, predominando sintomatologia osteoarticular como dorsolombalgia $(n=10)$, seguida por tumefação óssea $(n=6)$, e cifose $(n=4)$. A tuberculose miliar manifestou-se por febre prolongada $(n=$ 8) e na meningite tuberculosa predominaram os sintomas neurológicos ( $n=6)$ e a febre $(n=2)$. Na forma intestinal e/ ou peritoneal a clínica era inespecífica com astenia $(n=4)$, perda ponderal $(n=3)$, febre $(n=4)$, dor abdominal $(n=3)$ e ascite $(n=1)$. Na tuberculose pleural registou-se tosse ( $n$ $=3)$, febre $(n=3)$ e toracalgia $(n=1)$. Na única doente com tuberculose renal, o sinal de alerta foi a hematúria com astenia e emagrecimento. A forma cutânea manifestou-se por úlcera crónica da face, com evolução de dois anos e sem melhoria após vários antimicrobianos. As formas raras de doença da parede torácica e glândulas salivares cursaram com tumefações com evolução crónica e sem repercussão no estado geral dos doentes.

As formas graves observadas em 15/145 (10,3\%) doentes incluíram sete com meningite e oito com doença miliar. Destes, oito tinham sido vacinados com BCG e no que respeita ao grupo etário, dois apresentavam idade inferior a 12 meses, um três anos de idade e cinco eram adolescentes com mais de 13 anos. Um dos doentes com tuberculose

Tabela 3 - Caracterização da sintomatologia dos doentes com tuberculose extrapulmonar

\begin{tabular}{|c|c|}
\hline Tipo de TbEP $(n)$ & Sintomatologia $(n)$ \\
\hline Ganglionar (26) & $\begin{array}{l}\text { Febre (11) } \\
\text { Tosse (10) } \\
\text { Astenia (7) } \\
\text { Perda ponderal (7) } \\
\text { Adenopatia cervical de evolução sub-aguda/crónica (6) } \\
\text { Toracalgia (4) }\end{array}$ \\
\hline Óssea (15) & $\begin{array}{l}\text { Febre (8) } \\
\text { Dorsolombalgia (10) } \\
\text { Tumefação óssea (6) } \\
\text { Cifose (4) }\end{array}$ \\
\hline Miliar (8) & Febre (8) \\
\hline Meníngea (7) & $\begin{array}{l}\text { Sintomas neurológicos (6) } \\
\text { Febre (2) }\end{array}$ \\
\hline Peritoneal/intestinal (6) & $\begin{array}{l}\text { Astenia (4) } \\
\text { Perda ponderal (3) } \\
\text { Febre (4) } \\
\text { Dor abdominal (3) } \\
\text { Ascite (1) }\end{array}$ \\
\hline Pleural (4) & $\begin{array}{l}\text { Tosse (3) } \\
\text { Febre (3) } \\
\text { Toracalgia (1) }\end{array}$ \\
\hline Renal (1) & Hematúria com astenia e emagrecimento (1) \\
\hline Cutânea (1) & Úlcera crónica da face com evolução de dois anos, sem melhoria após vários antimicrobianos (1) \\
\hline Parede torácica (1) & Tumefação com evolução crónica (1) \\
\hline Glândulas salivares (1) & Tumefação com evolução crónica (1) \\
\hline
\end{tabular}


miliar apresentava infeção VIH.

O teste de sensibilidade à tuberculina (TST) foi positivo na maioria dos doentes [78/99 $(78,8 \%)]$, mas anérgico em 19/99 (19,2\%). O IGRA também foi positivo na maioria: $61 / 90(67,8 \%)$ dos doentes. O TST e o IGRA foram realizados simultaneamente em 54 doentes (37,2\%). Onze destes doentes tinham TST anérgico e IGRA negativo e um tinha TST e IGRA negativo. O IGRA-Quantiferon Gold Plus foi realizado como primeira escolha em 88/90 (97,8\%), tendo resultado positivo em 52/88 (59,1\%). Dos doentes que realizaram IGRA-Quantiferon, 35,2\% tinham menos do que cinco anos de idade e 15,9\% tinham menos de dois anos. Destes, o teste foi positivo apenas em $54,8 \%$ e $57,1 \%$, respectivamente. O IGRA T-SPOT foi realizado em 24/90 (26,7\%): em doentes com IGRA-Quantiferon indeterminado ( $n=6)$, com IGRA-Quantiferon negativo $(n=11)$, como primeira escolha $(n=7)$, tendo sido positivo em nove $(37,5 \%)$. Os casos positivos ocorreram em $5 / 8$ doentes com menos de cinco anos e em um com menos de dois anos de idade.

$\mathrm{Na}$ TP, o padrão da radiografia torácica mostrou condensação $(n=24)$, cavitação $(n=20)$, adenopatias hilares $(n=15)$, infiltrados nodulares $(n=9)$ e derrame pleural $(n=$ 5). A tomografia computorizada (TC) torácica realizada em
72/76 doentes confirmou e diagnosticou de novo condensação $(n=46)$, adenopatias hilares $(n=35)$, cavitação $(n=$ 31 ), imagens parenquimatosas nodulares $(n=21)$, derrame pleural $(n=8)$, calcificações $(n=7)$ e atelectasia $(n=5)$. Em cinco doentes em que a radiografia torácica foi considerada normal, a TC torácica identificou alterações, nomeadamente imagens nodulares parenquimatosas, condensação e calcificações, ajudando a estabelecer o diagnóstico. A broncofibroscopia realizada em 41/76 (53,9\%) crianças estava alterada em $73,2 \%$ com aspecto inflamatório inespecífico $(n=22)$ e granuloma endobrônquico $(n=8)$.

Registaram-se complicações em 24 (16,6\%) doentes. $\mathrm{Na}$ doença pulmonar parenquimatosa e com cavitações: derrame pleural $(n=8)$, fístula broncopleural $(n=1)$, lesão do palato duro $(n=1)$ e amiloidose secundária $(n=1)$. $\mathrm{Na}$ TbEP: cifose $(n=6)$, escoliose $(n=1)$, protusão lombar $(n=1)$, abcesso paravertebral $(n=1)$, síndrome de ativação macrofágica $(n=1)$, acidente vascular isquêmico da artéria cerebral média esquerda $(n=1)$, epilepsia $(n=1)$, agravamento da epilepsia de base $(n=1)$. Um ano após o diagnóstico registaram-se sequelas em 12 doentes (8,3\%): cifose $(n=6)$, escoliose $(n=1)$, hemiparesia direita e atraso do desenvolvimento $(n=1)$, epilepsia com polineuropatia

Tabela 4 - Comparação entre doentes com tuberculose pulmonar e tuberculose extrapulmonar

\begin{tabular}{|c|c|c|c|c|}
\hline & TB & TP & TbEP & Valor-p \\
\hline Idade (mediana; anos) & 12,9 & 14,6 & 10 & 0,006 \\
\hline Grupo etário & $\begin{array}{l}0-12 \text { meses } \\
1-5 \text { anos } \\
5-10 \text { anos } \\
>10 \text { anos }\end{array}$ & $\begin{array}{l}6 / 76(7,9 \%) \\
13 / 76(17,1 \%) \\
4 / 76(5,3 \%) \\
53 / 76(69,7 \%)\end{array}$ & $\begin{array}{l}5 / 69(7,2 \%) \\
18 / 69(26,1 \%) \\
12 / 69(17,4 \%) \\
34 / 69(49,3 \%)\end{array}$ & $\begin{array}{l}0,883 \\
0,188 \\
\mathbf{0 , 0 2 0} \\
\mathbf{0 , 0 1 2}\end{array}$ \\
\hline Nacionalidade & $\begin{array}{l}\text { Portuguesa } \\
\text { Não portuguesa }\end{array}$ & $\begin{array}{l}43 / 76(56,6 \%) \\
33 / 76(43,4 \%)\end{array}$ & $\begin{array}{l}32 / 69(46,4 \%) \\
37 / 69(53,6 \%)\end{array}$ & $\begin{array}{l}0,22 \\
0,22\end{array}$ \\
\hline BCG & $108 / 145(74,5 \%)$ & $61 / 76(80,3 \%)$ & $47 / 69(68,1 \%)$ & 0,094 \\
\hline Doença crónica & $29 / 145(20,0 \%)$ & $18 / 76(23,7 \%)$ & $11 / 69(15,9 \%)$ & 0,244 \\
\hline $\begin{array}{l}\text { TST } \\
\text { Realizado } \\
\text { Positivo } \\
\text { Negativo } \\
\text { Anérgico }\end{array}$ & $\begin{array}{l}99 / 145(68,3 \%) \\
78 / 99(78,8 \%) \\
2 / 99(2,0 \%) \\
19 / 99(19,2 \%)\end{array}$ & $\begin{array}{l}44 / 53(83,0 \%) \\
1 / 53(1,9 \%) \\
8 / 53(15,1 \%)\end{array}$ & $\begin{array}{l}34 / 46(73,9 \%) \\
1 / 46(2,2 \%) \\
11 / 46(23,9 \%)\end{array}$ & $\begin{array}{l}0,269 \\
0,919 \\
0,266\end{array}$ \\
\hline $\begin{array}{l}\text { IGRA Quantiferon } \\
\text { Realizado } \\
\text { Positivo } \\
\text { Negativo } \\
\text { Indeterminado }\end{array}$ & $\begin{array}{l}88 / 145(60,7 \%) \\
52 / 88(59,1 \%) \\
26 / 88(29,5 \%) \\
10 / 88(11,4 \%)\end{array}$ & $\begin{array}{l}27 / 41(65,9 \%) \\
9 / 41(22,0 \%) \\
5 / 41(12,2 \%)\end{array}$ & $\begin{array}{l}25 / 47(53,2 \%) \\
17 / 47(36,2 \%) \\
5 / 47(10,6 \%)\end{array}$ & $\begin{array}{l}0,228 \\
0,145 \\
0,818\end{array}$ \\
\hline $\begin{array}{l}\text { IGRA T-SPOT } \\
\text { Realizado } \\
\text { Positivo } \\
\text { Negativo }\end{array}$ & $\begin{array}{l}24 / 145(16,6 \%) \\
9 / 24(37,5 \%) \\
15 / 24(62,5 \%)\end{array}$ & $\begin{array}{l}1 / 11(9,1 \%) \\
10 / 11(90,9 \%)\end{array}$ & $\begin{array}{l}8 / 13(61,5 \%) \\
5 / 13(38,5 \%)\end{array}$ & $\begin{array}{l}0,008 \\
0,008\end{array}$ \\
\hline Agente identificado & $86 / 145(59,3 \%)$ & $51 / 76(67,1 \%)$ & $35 / 69(50,7 \%)$ & 0,045 \\
\hline TB bacilífera & $30 / 145(20,7 \%)$ & $26 / 76(34,2 \%)$ & $4 / 69(5,8 \%)$ & $<0,001$ \\
\hline Mediana do internamento (dias) & 15 & 12,5 & 22 & 0,011 \\
\hline Mediana da terapêutica (meses) & 6 & 6 & 9 & $<0,001$ \\
\hline Complicações & $24 / 145(16,6 \%)$ & $11 / 76(14,5 \%)$ & $13 / 69(18,8 \%)$ & 0,480 \\
\hline Sequelas & $12 / 145(8,3 \%)$ & $1 / 76(1,3 \%)$ & $11 / 69(15,9 \%)$ & 0,001 \\
\hline Total & 145 & 76 & 69 & \\
\hline
\end{tabular}


dos VI e VII pares $(n=1)$, epilepsia refratária e atraso do desenvolvimento $(n=1)$, lobectomia por granulomas $(n=1)$ e fibrose pulmonar $(n=1)$.

Houve identificação do agente em 86 doentes (59,3\%). O exame direto foi positivo em $21,4 \%$, o teste de amplificação de ácidos nucleicos (TAAN) em $47,6 \%$ e o exame cultural em $59,3 \%$. O agente foi identificado no suco gástrico $(n=48)$, expetoração $(n=18)$, lavado broncoalveolar $(n=$ 14 ) e em outros locais $(n=18)$. Em 107 doentes $(73,8 \%)$ o agente foi pesquisado em mais do que um local e identificado em simultâneo em diferentes amostras em 20 casos $(13,8 \%)$.

Em 30 doentes $(20,7 \%)$ identificaram-se bacilos ácido-álcool resistentes em exame direto no suco gástrico/expetoração. Dezasseis doentes (11\%) tinham resistências a antibacilares isoladamente com isoniazida ( $n=9)$ ou estreptomicina $(n=3)$, e a isoniazida com mais um fármaco com estreptomicina $(n=2)$, etambutol $(n=1)$ e pirazinamida $(n=1)$. Em 2/16 existia história prévia de TB latente com terapêutica com isoniazida.

Em 11\% $(n=16)$ registaram-se efeitos secundários da terapêutica antibacilar com hiperuricemia $(n=9)$, hepatite tóxica $(n=6)$, polineuropatia axonal sensitiva $(n=1)$ e lúpus induzido por fármacos $(n=1)$, sendo que um doente apresentou duas complicações (hiperuricemia e lúpus induzido por fármacos). Estas complicações ocorreram em oito doentes com doença crónica. Nos casos de hepatite tóxica a mediana de dias até ao aparecimento de hepatite foi de 21 dias $(5-60)$. A interrupção da terapêutica foi feita em quatro doentes até melhoria das transaminases. Um doente manteve a terapêutica porque a elevação das transaminases era ligeira, tendo mantido monitorização laboratorial. Nos casos de hiperuricemia, seis doentes iniciaram terapêutica com alopurinol. O doente com lupus induzido por fármacos suspendeu a isoniazida, tendo mantido rifampicina e levofloxacina. $O$ doente com polineuropatia axonal foi medicado com piridoxina em dose terapêutica de neuropatia.

A mortalidade foi de $0,7 \%$, com um óbito em uma doente evacuada de PALOP com neoplasia em fase avançada, desnutrição crítica e que se encontrava em cuidados paliativos.

A comparação entre os doentes com TP e TbEP pode ser avaliada na Tabela 4.

Comparámos a TP e TbEP e verificámos que na TP o agente foi mais frequentemente identificado $(67,1 \%$ vs $50,7 \% ; p=0,045)$ e os doentes eram mais frequentemente bacilíferos $(34,2 \%$ vs $5,8 \% ; p<0,001)$. Os doentes com TbEP eram mais jovens (mediana 10 vs 14,6 anos; $p=$ $0,006)$, com maior número de TST anérgicos, mas sem diferença estatisticamente significativa $(23,9 \%$ vs $15,1 \%$; $p=$ $0,266)$. Os testes com resultados anérgicos ocorreram em doentes com meningite tuberculosa, idade inferior a dois anos e na terapêutica com corticoterapia. A duração do internamento foi mais prolongada na TbEP (mediana 22 vs 12,5 dias; $p=0,011$ ), assim como a duração da terapêutica (mediana nove versus seis meses; $p<0,001$ ). Os interna- mentos foram mais prolongados na TB óssea (mediana 27 dias), miliar (mediana 29 dias) e meníngea (mediana 31 dias). Verificou-se ainda maior duração do internamento em doentes bacilíferos (mediana 27,5 vs 13,5 dias; valor- $p$ $=0,004)$.

\section{DISCUSSÃO}

Apesar de rara em países desenvolvidos, a incidência da TB em Pediatria não é negligenciável e cursa com morbilidade importante..$^{1-3}$ Os casos de TB em doentes pediátricos nascidos em Portugal, cujo caso índex não foi identificado, correspondem a casos de TB bacilífera na comunidade não diagnosticados. ${ }^{5,6} \mathrm{Em} \mathrm{2017}$, foi reportada uma incidência de TB de 6,1 casos por 100000 crianças até aos cinco anos de idade, mas os dados sobre a doença na população pediátrica são escassos. ${ }^{12}$ Embora as crianças constituam $10 \%$ a $20 \%$ do peso global da TB, têm sido cronicamente negligenciadas. ${ }^{13-15} \mathrm{~A}$ ausência de critérios de diagnóstico estandardizados, a variabilidade do quadro clínico, e o facto de frequentemente não serem bacilíferas com culturas negativas, levam às dificuldades inerentes para se realizar o diagnóstico, ${ }^{13,16}$ que neste grupo exige um elevado grau de suspeição. $O$ facto das crianças terem maior risco de doença grave, e da infeção na infância contribuir para reservatório de novos casos no futuro, ${ }^{9}$ mostra que esta entidade não deve ser negligenciada.

A maioria dos doentes deste estudo era natural de Portugal, ao contrário de outros estudos realizados em países desenvolvidos onde a população é maioritariamente imigrante. ${ }^{16,17} \mathrm{O}$ estudo mostra que, na maioria, as crianças foram infectadas nos seus agregados familiares, mas que apesar da doença ser conhecida no adulto, o tempo que mediou entre o diagnóstico no caso índex e nos nossos doentes teve uma média superior a um ano (13,7 meses). Por outro lado, apesar de 50 doentes terem um contacto com TB conhecido, apenas 29 foram rastreados. Estes aspectos sugerem que o rastreio dos contactos de doentes com TB poderá estar muito aquém do desejado, podendo necessitar de reformulações e melhorias.

O aumento do número de casos em 2017 relaciona-se em parte com um surto de um caso de TB bacilífera de um adulto, com tempo alargado entre o início dos sintomas e o diagnóstico, ${ }^{12}$ mas também com falhas na identificação da doença em grupos de risco. Por outro lado, a estabilização do número de doentes oriundos de PALOP, onde a TB é endémica, alerta para a importância da realização de um rastreio sistemático nesta população.

A retirada da vacina $B C G$ do Programa Nacional de Vacinação para a população em geral no ano de 2016 não teve qualquer influência no número de casos neste estudo. A maioria dos doentes tinha vacina BCG, que como é sabido não tem qualquer interferência na transmissão da doença pulmonar, mas neste estudo também não preveniu formas graves como a meningite tuberculosa e TB miliar em doentes saudáveis ( $n=8$; cinco doentes com TB meníngea e três com TB miliar), confirmando a necessidade de novas vacinas com impacto na imunidade de grupo. ${ }^{18}$ 
O diagnóstico apresentou-se como um desafio, nomeadamente pela ausência de especificidade dos sintomas, dificuldades em obter confirmação bacteriológica e pela elevada ocorrência de doença extrapulmonar. ${ }^{13,16,19}$

A clínica, tal como está descrito na literatura, foi inespecífica, com sintomas comuns como tosse, febre, astenia e perda ponderal. ${ }^{17,19,20} \mathrm{O}$ agente identificado em $59,3 \%$ dos casos ocorreu principalmente na TP, como acontece na idade adulta. Esta percentagem foi sobreponível ao encontrado noutros estudos. ${ }^{16,17}$ No entanto, a percentagem de doentes com exame cultural positivo foi superior $(59,3 \%$ vs $25 \%$ - 50\%), ${ }^{9,13}$ o que pode indicar uma boa capacidade técnica do laboratório de microbiologia do nosso hospital.

O TST e o IGRA podem auxiliar no diagnóstico mas não distinguem a doença ativa da infeção latente, sendo fundamental a sua associação à clínica, achados radiológicos e contexto epidemiológico. ${ }^{21} \mathrm{O}$ TST foi positivo em $78,8 \%$ e ligeiramente superior a outros estudos $\left(72,3 \%{ }^{21}\right.$ e $\left.53,3 \%\right) .{ }^{19}$ O IGRA poderá ser mais benéfico no contexto de TB latente. ${ }^{22}$ Neste estudo, a positividade do IGRA-Quantiferon e do T-SPOT foi inferior a outros descritos na literatura, mas não foram realizados em todos os doentes, o que poderá condicionar os resultados. ${ }^{22}$

As formas de doença extrapulmonar apresentam uma maior complexidade e exigem uma forte suspeição clínica para o diagnóstico. A taxa de 47,6\% identificada deste estudo foi mais elevada que a reportada em outros países como a Turquia $(35 \%)^{19}$ e Dinamarca $(24,8 \%)^{16}$ mas, tal como descrito na literatura, foi mais frequente em imigrantes e em doentes mais jovens. ${ }^{16,19}$

No mundo morrem anualmente cerca de 210000 crianças por complicações de $\mathrm{TB},{ }^{23} \mathrm{com}$ a maior taxa de mortalidade e de sequelas associada à meningite tuberculosa. ${ }^{9}$ Neste estudo, $16,6 \%$ dos casos tiveram complicações e $8,3 \%$ sequelas, sendo que três doentes apresentaram complicações e sequelas associadas ao envolvimento do sistema nervoso central. Também o número considerável de sequelas em doentes com TB óssea (7/15; 46,7\%) confirma que a morbilidade da doença se mantém elevada mesmo em países com bons recursos de tratamento.

A resistência à terapêutica antibacilar em $11 \%$ dos doentes é significativa, e justifica de acordo com o nosso parecer uma monitorização e vigilância apertadas, em consonância com outros estudos. Sobre a resistência em idade pediátrica sabe-se que num estudo feito na Dinamarca a resistência foi de $11 \%,{ }^{16}$ em Espanha sabe-se que a prevalência estimada é superior a $4 \%{ }^{24}$ e na Europa a taxa de TB multirresistente é de $3,7 \%$ (> 10\% dos casos reportados na Estónia e Lituânia). ${ }^{25}$

O presente estudo é mais abrangente que outros estudos realizados em Portugal ${ }^{26}$ e revela uma elevada morbilidade e consequente interferência na saúde da criança. Estes aspectos deverão ser objeto de reflexão séria com o propósito de implementar medidas que visem a diminuição da doença pediátrica no nosso país.

O estudo apresenta algumas limitações, nomeadamente o facto de ser unicêntrico e retrospectivo, baseado na colheita de dados de processos clínicos, existindo a possibilidade de parte da informação se encontrar omitida.

\section{CONCLUSÃO}

A TB é pouco frequente em Pediatria, mas tem um impacto significativo na saúde da criança, pelo que não deve ser negligenciada. As formas extrapulmonares são mais frequentes neste grupo etário, apresentam maior complexidade de diagnóstico e tratamento e exigem uma forte suspeição clínica para o seu diagnóstico.

$\mathrm{Na}$ criança é fundamental diminuir o tempo até à realização do diagnóstico e assim prevenir as formas graves, mas também, e não menos importante, no adulto, para se conseguir diminuir o período de contagiosidade. $O$ estudo alerta ainda para a importância do rastreio sistemático da infeção em crianças de PALOP, em imunocomprometidos e com doença crónica.

\section{OBSERVAÇÕES}

Dados parciais apresentados em comunicação oral nas $11^{\text {as }}$ Jornadas de Atualização em Doenças Infeciosas do Hospital de Curry Cabral do CHULC, realizadas nos dias 25 e 26 de janeiro de 2018, Lisboa.

Poster nas $12^{\text {as }}$ Jornadas de Atualização em Doenças Infeciosas do Hospital de Curry Cabral do CHULC, realizadas nos dias 23 e 24 de janeiro de 2020, Lisboa, com o Prémio de Melhor Poster do Congresso.

\section{CONTRIBUTO DOS AUTORES}

MB: Desenho do artigo, armazenamento e recolha de dados, participação na análise e interpretação de dados, redação do manuscrito, revisão e discussão dos resultados.

APR: Desenho do artigo, recolha de dados, participação na análise e interpretação de dados.

CVM: Recolha de dados, participação na análise e interpretação de dados.

TMS: Recolha de dados, participação na análise e interpretação de dados, na revisão de versões e revisão crítica do conteúdo.

CG, FC: Participação na revisão de versões e revisão crítica do conteúdo.

MJB: Desenho do artigo, participação na revisão de versões, revisão crítica do conteúdo e aprovação da versão final.

\section{PROTEÇÃO DE PESSOAS E ANIMAIS}

Os autores declaram que os procedimentos seguidos estavam de acordo com os regulamentos estabelecidos pelos responsáveis da Comissão de Investigação Clínica e Ética e de acordo com a Declaração de Helsínquia da Associação Médica Mundial atualizada em 2013.

\section{CONFIDENCIALIDADE DOS DADOS}

Os autores declaram ter seguido os protocolos do seu centro de trabalho acerca da publicação de dados. 


\section{CONFLITOS DE INTERESSE}

Os autores declaram não ter conflitos de interesses relacionados com o presente trabalho.

\section{REFERÊNCIAS}

1. World Health Organization. Global tuberculosis report 2018. Geneva: WHO; 2018.

2. World Health Organization. Global tuberculosis report 2019. Geneva: WHO; 2019.

3. World Health Organization. Global tuberculosis report 2020. Geneva: WHO; 2020.

4. Direção-Geral da Saúde. Estratégia de vacinação contra a tuberculose com a vacina BCG, NORMA Nº06/2016. Lisboa: DGS; 2016.

5. Direção-Geral da Saúde. Programa nacional para a infeção VIH, SIDAe Tuberculose. Lisboa: DGS; 2015.

6. Direção-Geral da Saúde. Programa nacional para a infeção VIH, SIDAe Tuberculose. Lisboa: DGS; 2017.

7. Kimberlin DW, Brady MT, Jackson MA, Long SS, editors. Red Book: 2015 Report of the Committee on Infectious Diseases. 30 $0^{\text {th }}$ ed. Elk Grove Village: American Academy of Pediatrics; 2015.

8. Kritsaneepaiboon S, Andres MM, Tatco VR, Lim CC, Concepcion ND. Extrapulmonary involvement in pediatric tuberculosis. Pediatr Radiol. 2017; $47: 1249-59$.

9. Cruz AT, Starke JR. Pediatric tuberculosis. Pediatr Rev. 2010;31:13-26.

10. Thomas TA. Tuberculosis in children. Pediatr Clin N Am. 2017;64:893909.

11. Newton SM, Brent AJ, Anderson S, Whittaker E, Kampmann B. Pediatric tuberculosis. Lancet Infect Dis. 2008;8:498-510.

12. Direção Geral da Saúde. Programa Nacional para a Tuberculose, Tuberculose em Portugal, Desafios e Estratégias. Lisboa: DGS; 2018.

13. Perez-Velez CM. Pediatric tuberculosis: new guidelines and recommendations. Curr Opin Pediatr. 2012;24:319-28.

14. Tsai KS, Chang HL, Chien ST, Chen KL, Chen KH, Mai MH, et al. Childhood tuberculosis: epidemiology, diagnosis, treatment, and vaccination. Pediatr Neonatol. 2013;54:295-302.

15. Burki T. Neglecting childhood tuberculosis "a human rights violation".

\section{FONTES DE FINANCIAMENTO}

Este trabalho não recebeu qualquer tipo de suporte financeiro de nenhuma entidade no domínio público ou privado.

Lancet Infect Dis. 2018;18:723.

16. Hatleberg $\mathrm{Cl}$, Prahl JB, Rasmussen JN, Andersen $\mathrm{PH}$, Bjerrum S, Thomsen $\mathrm{V}$, et al. A review of paediatric tuberculosis in Denmark: 10 year trend, 2000-2009. Eur Respir J. 2014;43:863-71.

17. Kitai I, Morris SK, Kordy F, Lam R. Diagnosis and management of pediatric tuberculosis in Canada. CMAJ. 2017;189:E11-6.

18. Chawla S, Garg D, Jain R, Khanna P, Choudhary S, Sahoo S, et al. Tuberculosis vaccine - time to look into future. Hum Vaccin Immunother. 2013;10:420-2.

19. Turel O, Kazanci S, Gonen I, Aydogmus C, Karaoglan E, Siraneci R. Paediatric tuberculosis at a referral kospital in Istanbul: analysis of 250 Cases. Biomed Res Int. 2016;2016:6896279:1-6.

20. Yunda L, Sepúlveda E, Herrera KC, Moreno C. Pulmonary tuberculosis in a pediatric reference hospital in Bogotá, Colombia. Int J Mycobacteriol. 2017;6:258-63.

21. Cano A, Romaneli M, Pereira R, Tresoldi A. Tuberculosis in pediatric patients: how has the diagnosis been made? Rev Paul Pediatr. 2017;35:165-70.

22. Gaensbauer J, Broadhurst R. Recent innovations in diagnosis and treatment of pediatric tuberculosis. Curr Infect Dis Rep. 2019;21:4.

23. Lamb GS, Starke JR. Tuberculosis in infants and children. Microbiol Spectrum. 2017;5:2:TNMI7-0037-2916.

24. Peña MJ, García BS, Baquero-Artigao F, Pérez DM, Pérez RP, Echevarría AM, et al. Actualización del tratamento de la tuberculosis em niños. An Pediatr. 2018;88:52:e1-52.e12.

25. European Centre for Disease Prevention and Control. Tuberculosis surveillance and monitoring in Europe 2020 - 2018 data. Stockholm: ECDC; 2020.

26. Gonçalves J, Cerqueira A, Machado C, Carvalho F, Cruz S, Gonçalves A, et al. Tuberculose em idade pediátrica: características, incidência e distribuição geográfica (2000-2010). Acta Pediatr Port. 2012;43:104-10. 